\title{
PROKLA-Redaktion
}

\section{Editorial: Ökonomie des Konsums}

1.

... durch den Luxus finden

Millionen Armer sich erhalten.

Auch durch den Stolz, den alle schalten.

Nicht minder dient der Neid sowie

die Eitelkeit der Industrie.

Die Sucht, sich als modern in Speisen, in Kleid und Möbeln zu erweisen, stets ein Objekt des Spottes zwar, des Handels wahre Triebkraft war.

... so schrieb Bernard Mandeville 1705 in seiner Bienenfabel und führte damit vor genau 300 Jahren eine Argumentationsfigur ein, die den Konsum der Wohlhabenden in ein damals gänzlich neues Licht tauchte. Tatsächlich erfolgten im Lauf des 18. Jahrhunderts Veränderungen im Alltagsleben, die Historiker bereits für diese Periode in England von einer „Konsumrevolution" sprechen lassen. Anzeichen dafür gab es im zunehmenden Verbrauch von Tee, dessen Import um das 200 fache stieg und und die nationale Sitte der teatime begründete, oder im Erfolg des Manufaktur-Unternehmers Josiah Wedgwood, der die besseren Kreise mit feinem weißen Porzellan und vielfältigsten $\mathrm{Mu}-$ stern versorgte. Wie Neil McKendrick festgestellt hat, wurden aus früheren „Luxuswaren“ nunmehr „Annehmlichkeiten“ und aus diesen bald „Notwendigkeiten“. Bis dahin hatten soziale oder religiöse Normen für die einzelnen Stände geregelt, was ihnen an Unentbehrlichem oder an Überflüssigem zustand. Nur für die oberste Schicht der Gesellschaft galten Prunk und Verschwendung als angemessen. Die Standesunterschiede sollten sich in der
Lebensführung abbilden und die mittleren und niedrigeren Schichten vor den Versuchungen einer leichtsinnigen Lebensführung bewahrt werden: indem man vorschrieb, wer Samt und Seide oder Schnabelschuhe tragen durfte oder aber wie viele Gäste höchstens bei einer Hochzeit einzuladen waren.

Zwar dehnten sich kapitalistische Lohnarbeit und Geldwirtschaft aus, doch musste die große Masse der Bevölkerung genügsam bleiben. Viele puritanische Unternehmer begrenzten nicht nur die Kaufkraft ihrer Arbeiter durch niedrige Löhne, sondern hingen auch selbst den Idealen der Sparsamkeit und der Askese an. Ihre Gewinne investierten sie eher ins eigene Geschäft als sie für Luxus und Tand auszugeben. Theateraufführungen erschienen ihnen als frivole Vergnügungen, kostbare Kleidungsstücke als Zeichen verwerflicher Eitelkeit. Der Autor der Bienenfabel wurde von seinen Zeitgenossen daher heftig getadelt, er würde zur „Lasterhaftigkeit“ ermutigen, wogegen er sich verwahrte. Er wolle lediglich beweisen, dass die immer neuen Bedürfnisse der Reichen Gewerbe und Handel belebten: „Private Vices, Publick Benefits". An eine Ausweitung dieser Bedürfnisspiralen auf die ärmeren Schichten, auf die Arbeiterschaft in Werkstätten und Manufakturen, dachte Mandeville gerade nicht: Er sprach ganz offenherzig aus, dass die Zahl der Armen, die mit ihrer Arbeit all diese Annehmlichkeiten produzierten, den wahren Reichtum einer Gesellschaft ausmachen. Was Mandeville in die öffentliche Diskussion ein- 
geführt hatte, war eine neue Legitimationsebene: die ökonomische Nützlichkeit anstelle des sittlich Gebotenen.

Damaligen wie späteren Autoren schien damit eine neue Freiheit (die man begrüßen oder fürchten konnte) anzubrechen. Der Konsum hatte sich seiner sozialen und moralischen Fesseln entledigt. Die Idee eines trickle down dieses Konsums wurde dann von Adam Smith 1759 in seiner Theory of Moral Sentiments formuliert. Gerade durch die selbstsüchtige Verfolgung ihrer Bedürfnisse verschaffen die Reichen den Armen Beschäftigung und Einkommen. Dass es sich dabei weniger um eine wissenschaftliche Beschreibung realer Vorgänge als um eine Apologie der bestehenden $\mathrm{Zu}-$ stände handelte, machte seine unmittelbar folgende Behauptung klar: In diesem Prozess würden die Reichen nämlich von einer „unsichtbaren Hand“ (von der hier zum ersten Mal die Rede ist) zu einer Verteilung der Lebensnotwendigkeiten geführt, die nur geringfügig anders aussieht, als wenn die Erde zu gleichen Teilen unter ihre Einwohner aufgeteilt worden wäre. Immerhin wurde damit aber der Anspruch der unteren Schichten am wachsenden Konsum anerkannt. Ganz anders argumentierte dagegen Thomas Malthus einige Jahre später. Einerseits rechtfertigte er den ausufernden Konsum der nichtkapitalistischen Klassen (Aristokratie, Kirche) mit dem Argument, nur so könnte der wachsende Output der immer produktiveren Fabriken und Werkstätten abgesetzt und Krisen vermieden werden. Andererseits forderte er, den Konsum der ärmeren Schichten zu begrenzen. Dies wäre durchaus im eigenen Interesse der Armen: würde ihr Lebensstandard steigen, so würden sie sich in einem so hohen Maße vermehren, dass die Lebensmittelproduktion nicht mehr mithalten könnte und es $\mathrm{zu}$ einer „Überbevölkerung“ käme, die sich gerade auf jene Armen am schlimmsten auswirken würde.

Die Entwicklung des Kapitalismus folgte, zumindest was den Konsum der unteren
Klassen anging, in der ersten Hälfte des 19. Jahrhunderts eher der von Malthus als der von Smith gezeichneten Linie. Überlange Arbeitszeiten gingen mit Hungerlöhnen einher, was Marx und Engels im Kommunistischen Manifest zu der pathetischen Aussage führten, die Bourgeoisie sei „unfähig zu herrschen, weil sie unfähig ist, ihrem Sklaven die Existenz, selbst innerhalb seiner Sklaverei zu sichern". Im Kapital war von einer solchen absoluten Verelendungstheorie dann aber nicht mehr die Rede. Hier begründete Marx nicht nur, dass Kapitalgewinn und Äquivalententausch vereinbar sind, er zeigte mit seiner Theorie des „relativen Mehrwerts“, dass steigende Gewinne durchaus mit einem steigenden Realeinkommen der vom Kapital Beschäftigten einhergehen können: dann nämlich, wenn zwar die Löhne einen immer geringeren Teil des neu produzierten Wertes umfassen, zugleich aber die Produktivität steigt, so dass von diesen wertmäßig sinkenden Lohn eine zunehmende Menge an Gebrauchsgütern gekauft werden kann. Zugleich machte Marx jedoch die Kehrseite dieser schönen neuen kapitalistischen Welt des Konsums deutlich: die konkurrenzvermittelte Dynamik von Akkumulation und Produktivkraftentwicklung bringt durch ihre Tendenz zu Überproduktion und Überakkumulation nicht nur immer wieder Krisen hervor, auch in ihrem ganz normalen Funktionieren führt eine Produktionsweise, die als Zweck allein die Profitmaximierung kennt, zu einer zerstörerischen Vernutzung von Mensch und Natur.

2.

Der Weg zur Gesellschaft des Massenkonsums war auch sonst keineswegs so geradlinig, wie er meist dargestellt wird. Er bedeutete zwar gelegentlich die Popularisierung vormaliger Luxusgüter, häufiger aber noch die Zurückdrängung vieler Formen von Eigenversorgung. Seit der zweiten Hälfte des 19. Jahrhunderts gerieten die proletarischen Schichten in den am weitest entwickelten Ländern in 
den Sog der sich ausdehnenden Konsumwirtschaft. Die Lohnerhöhungen, die sie erkämpften, ermöglichten es ihnen, sich Nahrungsmittel, Kleidung und Möbel zu kaufen, das Leben in den Städten erzwang aber auch eine derart kommerzialisierte Versorgung. In den Haushalten der früheren ländlichen Arbeiterschaft hatte man noch Kleinvieh gehalten, einen Gemüsegarten angelegt und Kerzen oder Textilien für den eigenen Gebrauch gefertigt. Das enge Zusammenwohnen in den wachsenden Städten machte die Industriearbeiterschaft dagegen abhängig vom Angebot von Bäckern, Fleischern und Kolonialwarengeschäften, von Kleidermagazinen und Altwarenhändlern. Was über den Markt befriedigt wurde, ging für die meisten über das Nötigste nicht hinaus, doch trugen erste Ansätze von Massenfertigung dazu bei, bestimmte Produkte eher erschwinglich zu machen als diejenigen handwerklicher Produktion so im Fall von Schuhen. In den Haushalten blieben Reste der früheren Eigenproduktion erhalten: in Form der Hausarbeit sowie beim Reparieren, Umarbeiten und Wiederverwenden der Gebrauchsgüter des Alltags.

Daneben gehörten zahlreiche Massengüter des 20. Jahrhunderts noch nie zum Konsum der Reichen und Wohlhabenden, sondern wurden von Anfang an für die breite Massse entwickelt. Ein Beispiel dafür stellt die Margarine dar, die von vornherein als „Kunstbutter“ einen preiswerten Ersatz für die teure Butter darstellen sollte und vor allem in Arbeiterkreisen Deutschlands oder Englands beliebt war. Da Walöl bis in die 1930er Jahre einen wesentlichen Grundstoff für die Margarineherstellung bildete und die Absatzzahlen dieser frühen Markenprodukte steil anstiegen, waren manche Walarten bereits damals vom Aussterben bedroht. Ein anderes heutzutage weitverbreitetes Produkt, das ebenso wenig mit den Lebensgewohnheiten der Oberschicht zu tun hat, ist das T-Shirt, das vor dem Ersten Weltkrieg als Unterbekleidung bei der US-Navy eingeführt wurde und für den zivilen Gebrauch später durch Künstler, die sich als unangepasste ,junge Wilde“ verstanden - etwa James Dean oder Marlon Brando -, bekannt wurde. Genauso unaristokratisch wie unbürgerlich ist auch die Herkunft des Kaugummis, dessen Geschichte und Bedeutung in der Massenkultur des 20. Jahrhunderts Michael $R$. Redclift im vorliegenden Band nachgeht. Der Kaugummi wurde von den US-amerikanischen GIs in vielen Teilen der Welt verbreitet und zu einem Symbol von Entspannung und Vergnügen. Der ursprüngliche $\mathrm{Na}$ turgummi wurde von den Mayas in Yucatan gewonnen. Sowohl die massenhafte US-amerikanische Nachfrage danach, wie auch seine spätere Ersetzung durch synthetischen Gummi hatten auf deren Gemeinwesen erhebliche Auswirkungen.

Für einen Teil der Gebrauchsgüter, die heutzutage zur Grundausstattung der meisten deutschen Haushalte gehören, kann man demgegenüber tatsächlich von einem trickle down sprechen. Um 1900 entfalteten sich im mittleren und höheren Bürgertum neue Bedürfnisse und Konsummuster. Man aß besser, kleidete sich aufwändiger, richtete sich in Plüsch und historisierendem Zierrat ein, legte sich Klaviere zum häuslichen Musizieren $\mathrm{zu}$ und verreiste im Winter wie im Sommer. Die Konsumpioniere der gehobenen Kreise nutzten neue technische Konsumgüter wie das Telefon und das Automobil. In den 1920er Jahren kamen weitere dazu: Radio, Staubsauger und Kühlschrank versprachen Unterhaltung oder Bequemlichkeit. In Ländern wie Großbritannien und Deutschland waren sie aufgrund ihrer Preise allerdings nur den höheren Schichten zugänglich, während sie in den USA bereits damals auch von weniger Wohlhabenden gekauft werden konnten.

Massenproduktion wurde zur Voraussetzung des Massenkonsums und als Inbegriff der Verknüpfung beider gilt das von Henry Ford Anfang des 20. Jahrhunderts in den USA eingeführte Geschäftsmodell. Das Auto, ein bisher überwiegend hand- 
werklich hergestelltes Produkt, sollte nunmehr aus normierten Teilen bestehen und die Typenzahl auf einige wenige Standardformen reduziert werden. Die Produktion großer Serien erfolgte durch eine Reihe von Einzweckmaschinen. Für die Mehrzahl der Arbeitskräfte waren Fachkenntnisse, Erfahrung und Geschicklichkeit weitgehend entbehrlich, so dass kurzfristig angelernte Arbeiter eingesetzt werden konnten. Die Verkettung der Arbeitsschritte durch Fließbänder stellte eine naheliegende Konsequenz des im Höchstmaß mechanisierten Prozesses dar. Die Arbeiter wurden somit, wie Marx es einige Jahrzehnte zuvor für die englische Textilindustrie beschrieben hatte, Anhängsel der Maschine - eine Degradierung, die sich bei Ford letztlich nur vermittels erheblicher Lohnerhöhungen durchsetzen ließ (ein Zugeständnis, das Ford allerdings damit verband, dass der Lebenswandel der Arbeiter in deren Wohnungen durch Fabrikfürsorgerinnen penibel überwacht wurde). Die kombinierte Wirkung höherer Löhne und niedrigerer Verkaufspreise erlaubte es mittel- bis langfristig, dass auch Arbeiter in die Lage versetzt wurden, die von ihnen hergestellten Produkte selbst zu erwerben. Eine Karikatur in einer deutschen sozialdemokratischen Zeitschrift der 1920er Jahre brachte den neuen Zusammenhang auf den Punkt: Auf einem ersten Bild sah man Arbeiter, die zu Fuß in die Fabrik strömten; auf dem zweiten Arbeiter, die mit dem Auto dorthin fuhren. Der ironische Kommentar lautete: „Welch ein Fordschritt“. Tatsächlich war die neue Produktionsform alles andere als widerspruchs- und konfliktfrei, doch den meisten Angehörigen der unteren Schichten erschien sie als Möglichkeit des Zugangs zu einer verheißungsvollen Konsumwelt. Der Tauschhandel, der ihnen angeboten wurde, lautete: Akzeptiere inhaltsleere, fremdbestimmte und im Höchstmaß kontrollierte Arbeit, dann erhältst du die Chance, Güter zu erwerben, die bisher den Wohlhabenden und Reichen vorbehalten waren.
Diesem Muster folgte mit Citroën zunächst die französische Automobilindustrie, später trachtete auch das nationalsozialistische Deutschland danach, es mit einem eigenen Auto für den ,kleinen Mann“ zu kopieren. Der „KdF-Wagen“ stellte eines von Hitlers Lieblingsprojekten dar, aber das modernste Automobilwerk Europas, in dem ab 1938 jährlich 1,5 Mio. Fahrzeuge hergestellt werden sollten, lieferte alles in allem nicht mehr als 630 davon, da die Rüstungsproduktion bereits einige Monate nach der Eröffnung des Werkes absoluten Vorrang erhielt. Erst in den folgenden Jahrzehnten fand der „Käfer“ tatsächlich millionenfach Verbreitung und trug mit dem Ford Taunus oder dem Opel Kadett da$\mathrm{zu}$ bei, dass die Arbeiterschaft und die einfachen Angestellten des bundesdeutschen "Wirtschaftswunders" in dem Gefühl lebten, auch sie hätten Anteil am neuen Wohlstand. Im Nationalsozialismus hatte sich der Prozess des trickle down letztlich auf den „Volksempfänger“ beschränkt, in der Bundesrepublik weitete er sich zunächst auf die Waschmaschine und den Farbfernseher aus, dann auf das Brathähnchen im Wienerwald und die Pauschalreise nach Rimini, später auf den tiefgefrorenen Hummer bei Aldi und den All-inclusive-Aufenthalt in „Dom-Rep“. Der andere deutsche Staat, die DDR, war, wie Jörg Roesler in seinem Beitrag zeigt, keineswegs durchgehend die „Mangelgesellschaft“, als die sie viele bundesdeutsche Beobachter sehen wollten. Dennoch gelang es dem Regime nicht, ein eigenes Modell des „sozialistischen Massenkonsums" durchzusetzen, sondern es musste sich nolens volens damit abfinden, dass die Bevölkerung ihre Konsumwünsche stets an den westlichen Standards maß.

3.

Gerade die Massenproduktion von $\mathrm{Au}-$ tomobilen hat in den USA und in den westeuropäischen Ländern immer wieder als Modell für die „Demokratisierung des Konsums“ hergehalten. Doch ver- 
stellt dieser plakative Begriff den Blick auf die Veränderungen des Konsums mehr, als dass er sie erklären würde. Mit „Demokratisierung“ wird eine Analogie zum schrittweisen politischen Strukturwandel seit der Mitte des 19. Jahrhunderts und insbesondere nach 1919 hergestellt - in Deutschland etwa das allgemeine Wahlrecht für Männer und Frauen und die verfassungsmäßige Verankerung von Grundrechten wie dem Recht der freien Meinungsäußerung, der Demonstrationsfreiheit oder dem Recht, sich in Parteien und Verbänden zu organisieren. Die tatsächliche Mitsprache der Bürgerinnen und Bürger bleibt normalerweise weit hinter den propagierten Idealen zurück - nicht nur weil ihnen Informationen fehlen oder vorenthalten werden und Lobbygruppen ihren Einfluss geltend machen, sondern auch aufgrund der strukturellen Zwänge, die von der kapitalistischen Grundlage der Wirtschaft ausgehen.

Was aber hat es zu bedeuten, wenn der Konsum als „Bürgerrecht“ bezeichnet wird und die Konsumentensouveränität als grundlegendes Merkmal westlicher Gesellschaften angesehen wird? Zwar hat eine Befreiung von mittelalterlichen Luxusverboten oder Bekleidungsvorschriften stattgefunden, und in kapitalistischen Ländern gibt es auch keine zentralen Planungsinstanzen, die die Anzahl der zu produzierenden Küchenmixer oder Damenpullover festlegt. Nicht die Zugehörigkeit zu einem Stand oder die Nähe zur Planungsbürokratie entscheidet somit über die Konsummöglichkeiten, sondern die finanziellen Möglichkeiten. Dennoch bedeutet das nicht, dass Konsumenten generell darüber mitentscheiden würden, was und wie produziert wird. Ansätze dazu gab es und gibt es allerdings stets nur außerhalb des kapitalistischen Unternehmertums. So entstand etwa in England 1844 die erste Konsumgenossenschaft der „redlichen Pioniere von Rochdale“, deren Ziel es war, die Arbeiterschaft mit guten und günstigen Lebensmitteln $\mathrm{zu}$ versorgen ( $\mathrm{zu}$ oft hatte man ihnen bis dahin mit Gips vermischtes Mehl oder Nudeln mit hohem Kreideanteil verkauft). Ähnlich trafen sich Radiobastler in den Arbeiter-Radioklubs der 1920er Jahre, um DetektorEmpfänger zusammenzubauen, und viele von ihnen träumten gleichzeitig davon, ihr eigenes Programm zu gestalten - ein Anliegen, das unter anderem von Bertolt Brecht unterstützt wurde, als er 1927 in seiner „Radiotheorie“ verkündete: „Der Rundfunk wäre der denkbar großartigste Kommunikationsapparat des öffentlichen Lebens..., wenn er es verstünde, nicht nur auszusenden, sondern auch zu empfangen, also den Zuhörer nicht $\mathrm{zu}$ isolieren, sondern ihn in Beziehung zu setzen.“.

Eine tatsächliche Mitwirkung der Konsumenten, jenseits der Kaufentscheidung zwischen Omo und Persil, ist in der kapitalistischen Marktwirtschaft aber gerade nicht vorgesehen. Zwar möchten Firmen die Wünsche ihrer potentiellen Kunden möglichst genau kennen, doch interessant sind solche Wünsche für Unternehmen nur, insoweit sie sich gewinnbringend befriedigen oder in eine solche Richtung verändern lassen. So stellen auch technische Innovationen üblicherweise nicht eine Antwort auf zuvor geäußerte Mängel dar, sondern folgen einer Logik, die im wesentlichen durch den Tüftlergeist von Ingenieuren und die Vorgaben von Marketing-Strategen bestimmt wird. Dies erkannte bereits Walther Rathenau, ältester Sohn Emil Rathenaus und später Vorstandsmitglied der AEG. „Die Elektrizität“, schrieb er 1907 in einem Brief, konnte sich „nicht darauf beschränken, lediglich Produkte zu Markte tragen, sie musste ihre eigenen Bedürfnisse schaffen..." So handelte es sich ihm zufolge bei der angewandten Elektrotechnik „um eine Umgestaltung eines großen Teils aller modernen Lebensverhältnisse, die nicht vom Konsumenten ausging, sondern vom Produzenten organisiert und gewissermaßen aufgezwungen werden muste.“

Diese Umgestaltungsprozesse fanden und finden nicht nur in den als entwickelt 
geltenden Ländern statt, transnationale Konsumgüterunternehmen bemühen sich seit längerer Zeit, sie auch in weniger entwickelte Regionen oder in Schwellenländer $\mathrm{zu}$ tragen. Häufig handelt es sich darum, die einheimische Bevölkerung auf Marktprodukte umzulenken, die bisherige Formen der Subsistenzarbeit oder der lokalen Produktion ersetzen sollen. Besonders verhängnisvoll wirkte sich seit den 1970er Jahren das aggressive Marketing der Firma Nestlé für Milchpulver aus, das Mütter dazu brachte, auf das Stillen ihrer Kinder zu verzichten. Dieser Schritt gefährdete in zahllosen Fällen die Gesundheit der Babys und führte zu ihrem Tod, da die örtlichen Gegebenheiten oft nicht für die Verwendung von Milchpulver geeignet waren. Auch bei vielen anderen „westlichen“ Produkten setzen transnationale Unternehmen mittlerweile auf rural marketing, also darauf, die ärmere und ländliche Bevölkerung anzusprechen. Michaela Kehrer untersucht diese Vorgehensweisen am Beispiel von Ägypten. Dabei wird deutlich, dass dies nicht unbedingt zu einer mit der Globalisierung einhergehenden Vereinheitlichung von Symbolen und Leitbildern führen muss, vielmehr instrumentalisieren transnationale Unternehmen, um ihren Absatz zu steigern, gerade auch lokale Kulturen.

4.

Um sich gegenüber ihren Konkurrenten zu behaupten, müssen Unternehmen andauernd Neues auf den Markt werfen. Das kann zu tatsächlichen Innovationen führen - oder auch nur zu Scheininnovationen, also zu neuen Produkten, die keinen wirklich neuen Gebrauchswert aufweisen, wie dies immer wieder bei vielen Erzeugnissen der Pharmaindustrie oder auch der Lebensmittelindustrie (etwa im boomenden Sektor des functional food) der Fall ist. Nicht selten wird die geballte Marktmacht großer Unternehmen dazu eingesetzt, um Produkte auf den Markt zu bringen, die im besten Fall überflüssig, oftmals jedoch sogar schäd- lich sind - als ein Beispiel für viele seien Hormonpräparate für die weiblichen Wechseljahre genannt. Mit jahrzehntelangen Marketing-Bemühungen gelang es den Herstellern, bei Ärzten und Patientinnen eine neue Normalität zu konstruieren, indem der bisherige Ausnahmefall klimakterieller Beschwerden umetikettiert und der Gebrauch dieser Präparate in vielen westlichen Ländern allgemeiner Usus wurde. Studien, die auf gesteigerte Krebsrisiken hinwiesen, wurden ignoriert oder als unglaubwürdig hingestellt. Darüberhinaus können Firmen neue Märkte auch dadurch erschließen, dass sie neue Gruppen von Konsumenten ausfindig machen: in den letzten Jahrzehnten etwa Kinder und Jugendliche, die als Abnehmer von Süßigkeiten und Chips, von Spielzeug und Sammelbildern, von Handys und Alcopos angesprochen werden und denen die Firmen möglichst früh „Markenbewusstsein“ beibringen wollen. Seit den ersten Vorboten des Massenkonsums verhieß dieser ein Leben, in dem Mühsal und Kargheit abgeschafft würden. Er rief aber immer wieder auch kritische Stimmen auf den Plan, die unheilvolle Wirkungen vor allem bei den Konsumenten selbst sahen - sei es, dass sie durch modischen Aufwand bei der Kleidung zu Eitelkeit und Leichtfertigkeit verführt oder durch populäre Unterhaltungsformen von ihren „eigentlichen“ Bedürfnissen entfremdet würden. Manche dieser Argumente mögen heutzutage moralisierend erscheinen. Dass moderne Formen des Konsums Menschen in der Entfaltung ihrer Fähigkeiten und Möglichkeiten nicht nur fördern, sondern auch behindern können, ist jedoch kaum zu bestreiten. Darüberhinaus wurde inzwischen immer deutlicher, dass es in bestimmten Bereichen $\mathrm{zu}$ ganz unmittelbaren Schädigungen der Konsumenten kommen kann. Eine Reihe von Skandalen machte den hohen gesundheitlichen Preis deutlich, den industrialisierte Methoden der Viehzucht und des Pflanzenbaus für sie haben können (u.a. BSE, Schweinepest). Diese spektaku- 
lären Fälle werden von den Beteiligten aus der Agrarwirtschaft gerne als unglückliche Pannen in einem sonst klaglos funktionierenden System dargestellt. Doch wiederholen sie sich immer wieder und ergänzen die permanenten Probleme der modernen Landwirtschaft wie die hohen Pestizid-Anteile in Gemüse und Obst. Dazu kommt die Tendenz, unverarbeitete durch verarbeitete Lebensmittel zu ersetzen, was nicht nur bei Kindern und Jugendlichen immer häufiger zu Fehlernährung und Dickleibigkeit führt. Dagmar Vinz wirft in ihrem Aufsatz die Frage auf, in welcher Weise Massenproduktion und -konsum im Bereich der Ernährung zusammenhängen und wie sich die Rolle der privaten Haushalte dabei geändert hat.

Mit wie viel Ressourcenverbrauch der Konsum zu tun hat, ist den Produkten nicht unmittelbar anzusehen. Auch bei Dienstleistungen findet ein großer Teil der damit verbundenen Arbeit backstage dar, ist also dem Blick des Restaurantoder Musical-Besuchers entzogen. Die Werbung tut ein übriges, Konsumgüter als selbstverständliche Gegebenheiten zu präsentieren und ihre Herkunft möglichst zu vernebeln. Die Attraktivität des american way of life und seiner Ableger in anderen Ländern hat zur Folge, dass die Bewohner der reicheren Länder den weitaus größten Teil der Energie und Rohstoffe verbrauchen und zugleich für den allergrößten Teil der Schädigungen verantwortlich sind: Ihr Fleischhunger führt dazu, dass Regenwälder in Weideland für Rinder umgewandelt und Sojabohnen zu 95\% an das Vieh verfüttert werden; mit ihren Flugreisen sorgen sie für die global zunehmende Luftbelastung. Wie am Fall der Wale zu sehen war, die bereits in der ersten Hälfte des 20. Jahrhunderts für die Margarineproduktion beinahe ausgerottet wurden, ist diese Umgangsweise mit natürlichen Ressourcen nicht grundsätzlich neu - sie hat aber mittlerweile ganz andere und weitaus bedrohlichere Dimensionen erreicht.
Neben der Nutzung natürlicher Ressourcen beruht die massenhafte Produktion von Konsumgütern auf dem Einsatz menschlicher Arbeitskraft. Dies geschieht allerdings unter sehr unterschiedlichen Bedingungen. Stellt man die Automobilindustrie als typisches Beispiel hin, so führt dies in die Irre. Hier gab es für eine bestimmte - die „fordistische“ - Periode einen Kompromiss zwischen den Ansprüchen der Unternehmen an hochproduktive Arbeitskräfte und den Ansprüchen der Arbeiter an eine Entlohnung, die sie am wachsenden Wohlstand teilhaben ließ. Dieses Modell der Massenproduktion entfaltete seine größte Wirksamkeit dort, wo es mit wohlfahrtsstaatlichen Regelungen und einer keynesianischen Wirtschaftspolitik abgesichert wurde. Für weite Bereiche der Konsumgüterproduktion galten jedoch immer schon ganz andere Bedingungen. So wurden die neuen Luxusgüter des 18. Jahrhunderts, Tee und Zucker, von Sklaven auf großen überseeischen Plantagen hergestellt. Als die Bekleidungskonfektion gegen Ende des 19. Jahrhunderts einen großen Aufschwung erlebte, verdankte sich dieser in den europäischen Großstädten und in New York vor allem der Arbeit von Frauen, die in Heimarbeit oder in sweatshops Löhne erhielten, die weit unter denjenigen der männlichen Industriearbeiter in den damaligen Wachstumsindustrien (etwa im Eisen- und Stahlsektor) lagen. Inzwischen hat es technologische Veränderungen der Produktion gegeben und an die Seite der kleinbetrieblichen Produktion sind Weltmarktfabriken getreten. Ansonsten aber sind die Verhältnisse der damaligen Arbeiterinnen durchaus mit denjenigen vergleichbar, unter denen heutzutage in Freien Produktionszonen oder in anderen weniger entwickelten Ländern für die Märkte der westlichen Länder produziert wird. Wie sich unter diesen Bedingungen die internationale Beschaffung einerseits, die Markt- und Konzentrationsstrukturen andererseits verändern, untersuchen Antje Blöcker und Michael Wortmann in ih- 
rem Beitrag anhand des Einzelhandels für Bekleidung.

5.

Wirft man einen Blick hinter die Kulissen der glanzvollen Konsum-Inszenierungen verschiedener Zeiten, ist das Ergebnis ernüchternd. In den letzten Jahren ist zumindest festzustellen, dass Konsum in der politischen und wissenschaftlichen Öffentlichkeit zum Thema geworden ist. Nicht-Regierungsorganisationen haben darüber aufgeklärt, unter welchen ökologisch und sozial katastrophalen Bedingungen Sportschuhe und T-Shirts, Kaffee und Kakao in vielen Ländern hergestellt werden. Das hat zwar sicherlich nicht ein radikales Umschwenken der Kaufgewohnheiten bewirkt, aber fair produzierte und gehandelte Konsumgüter als Alternative stärker ins Bild gerückt und ihren (geringen) Anteil leicht steigen lassen. Es hat auch dazu geführt, dass die Stiftung Warentest erstmals derartige Kriterien in ihre Beurteilungen von Produkten aufgenommen hat. Des weiteren sehen sich große Unternehmen mehr und mehr genötigt, sich zumindest verbal auf Nachhaltigkeitsziele zu verpflichten. Selbst wenn manchen Jugendlichen die Auswahl zwischen 10.000 Klingeltönen für ihr Handy als Beleg dafür gilt, dass sie in der besten aller Konsumwelten leben, gibt es andere, die sich für einen buy-nothing-day stark machen. Und schließlich wurde unlängst auf dem Weltsozialforum ein Manifest verabschiedet, das eines der elementarsten Konsumgüter überhaupt zum Gegenstand hat: das Wasser. Die unterzeichnenden Künstler und Menschenrechtler fordern, den Zugang zu Trinkwasser zum Menschenrecht zu erklären und die Wasserversorgung in vielen Ländern nicht den Interessen von Konzernen auszuliefern.

Zudem ist in den letzten Jahren angesichts von Massenarbeitslosigkeit und stagnierenden oder sinkenden Realeinkommen selbst in Deutschland die Quantität des Konsums erneut in den Blick gerückt. Das anscheinend so freundliche Gesicht des Wirtschaftswunderkapitalismus ist längst verschwunden. Inzwischen ist auch eine rot-grüne Regierung bei Rezepten angekommen, die an die eingangs erwähnten Auffassungen von Thomas Malthus erinnern (die Wandlungen rotgrüner Politik untersuchen außerhalb des Heftschwerpunkts Stefan Beck und Christoph Scherrer). Die erhebliche Senkung des Spitzensteuersatzes zu Jahresbeginn wurde mit einer Stärkung des Konsums, der die Wirtschaft ankurbeln soll, begründet. Die gleichzeitig in Kraft tretenden Hartz IV-Regelungen, die die Abschaffung der Arbeitslosenhilfe, verschärfte Zumutbarkeitsregeln und für Arbeitslose den Zwang zur Übernahme von „1-Euro-Jobs“ vorsehen, wurden dagegen mit den leeren Staatskassen einerseits, einer Verstärkung der „Anreize“ zur Arbeitsaufnahme andererseits begründet. Wie einst Malthus ist offensichtlich auch die rot-grüne Bundesregierung der Auffassung, dass der Kapitalismus lediglich den Konsum der Reichen, nicht aber den der Armen benötigt. Den Armen soll es nicht zu gut gehen, denn nur dann haben sie ausreichend Anreize, den Reichtum der anderen zu produzieren. 


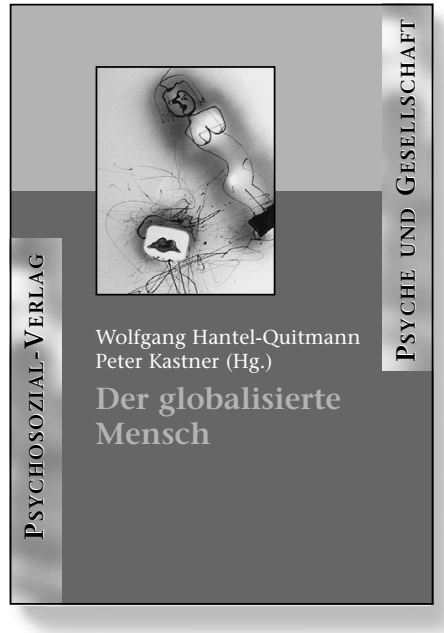

$2004 \cdot 284$ Seiten $\cdot$ Broschur EUR (D) 19,90 · SFr 34,90 ISBN 3-89806-289-9

Nach ihrem Buch Die Globalisierung der Intimität (Psychosozial-Verlag 2002) legen die Herausgeber eine neue Auswahl von Texten zur Globalisierung vor. Die Globalisierung ist nicht abstrakt, sie wirkt in vielen Bereichen sehr konkret in das Leben der Einzelnen hinein: Arbeit, Bildung, zwischenmenschliche Beziehungen, und viele andere.

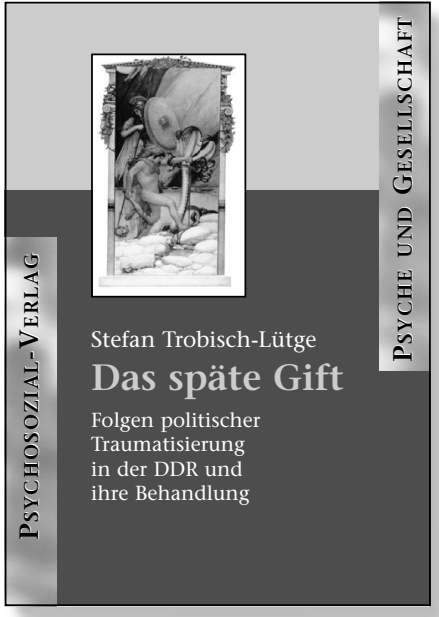

2004 - 171 Seiten · Broschur EUR (D) 19,90 · SFr 34,90 ISBN 3-89806-301- 1

Auch Jahre nach dem Ende der DDR lässt die Diktatur der SED viele Menschen nicht los. Noch heute tragen die politisch Verfolgten der DDR - der Gesellschaft weitgehend unbeachtet - zerstörerische psychische Implantate der Diktatur unbewusst weiter. Vor allem durch eine genaue Analyse der traumatisierenden Weitergabe seelischer Deformation wendet sich dieses Buch gleichermaßen an Betroffene und deren Angehörige, professionelle Helfer und interessierte Laien. 